\title{
Anti-angiogenic therapies for the treatment of angiosarcoma: a clinical update
}

\author{
Robin J. Young · Penella J. Woll
}

Received: 29 August 2017 / Accepted: 1 November 2017 / Published online: 7 November 2017 (C) The Author(s) 2017. This article is an open access publication.

\begin{abstract}
Summary Angiosarcomas are rare aggressive endothelial tumours, and are associated with a poor prognosis. Due to their vascular nature, there is great interest in their response to anti-angiogenic agents. A number of small prospective studies have reported angiosarcoma response to vascular-targeted agents, including agents that target vascular endothelial growth factor. To date, the response to these agents has been disappointing, and similar to the response observed in other soft tissue sarcoma subtypes. This short review will summarise the recent data in this field.
\end{abstract}

Keywords Angiosarcoma - Sarcoma - Anti-angiogenic therapy

\section{Introduction}

Angiosarcomas are rare aggressive endothelial tumours [1]. Angiosarcomas can occur spontaneously or secondary to radiotherapy, chronic lymphoedema and in a variety of familial conditions including neurofibromatosis (NF-1), mutated breast cancer associated gene 1 or 2 (BRCA1 or BRCA2) and Maffucci syndrome. They can develop at any site, but most commonly present as cutaneous disease, either as primary tumours on the head and neck of elderly white men, or on the chest wall secondary to breast cancer treatment. Complete excision with wide margins is often technically challenging due to disease extent and infiltration of surrounding tissue, effects of previous surgery/radiotherapy on local anatomy including lymphoedema, and co-morbidities in older

R. J. Young $(\bowtie) \cdot$ P. J. Woll

Academic Unit of Clinical Oncology, Weston Park Hospital, Whitham Rd, Sheffield, S10 2SJ, United Kingdom

r.j.young@sheffield.ac.uk patients. Angiosarcomas frequently recur despite surgery. Doxorubicin or paclitaxel are chemotherapies recommended for the treatment of recurrent/ advanced disease. Evaluation of new agents has been hampered by the rarity of angiosarcomas, but also by the difficulty in assessing response in diffuse infiltrating tumours. Hence progression-free survival (PFS) and overall survival (OS) are more robust endpoints than response rate.

Over the past 20 years, the tumour vasculature has become established as a target in the treatment of cancer, particularly with agents that target vascular endothelial growth factor (VEGF). As angiosarcomas are endothelial tumours, there is interest in the response of angiosarcoma to vascular-targeted agents. This short review will summarise the recent data in this field.

\section{Paclitaxel}

Paclitaxel is a microtubule-targeting chemotherapy with anti-angiogenic activity [2]. Response to weekly paclitaxel $\left(80 \mathrm{mg} / \mathrm{m}^{2}\right)$ was assessed in a prospective single arm phase II study of 30 patients with advanced angiosarcoma [3]; $36 \%$ of patients had received previous chemotherapy. The response rate (complete response $[\mathrm{CR}]+$ partial response $[\mathrm{PR}]$ ) was $19 \%$. Three patients with locally advanced disease had tumour responses that enabled subsequent surgery, and pathological CR were reported in 2 cases. The median PFS was 4 months, and the median OS was 7.4 months. Angiosarcoma responses to doxorubicin and paclitaxel have not been directly compared, but these results are similar to those obtained with anthracycline-based chemotherapy in 108 angiosarcoma patients prospectively enrolled in European Organisation for the Research and Treatment of Cancer (EORTC) trials, where the response rate was $25 \%$, 
with median PFS and OS of 4.9 and 9.9 months respectively, which were not significantly different to those in other soft tissue sarcomas (STS) [4]. Weekly paclitaxel is well tolerated and is often favoured as first-line therapy, particularly for the treatment of cutaneous angiosarcoma and in elderly patients.

\section{Agents targeting VEGF}

\section{Bevacizumab}

Bevacizumab is a humanised monoclonal antibody to VEGF-A. Angiosarcoma response to bevacizumab monotherapy $(15 \mathrm{mg} / \mathrm{kg} 3$ weekly) was studied in a single arm phase II study, which also recruited patients with epithelioid haemangioendothelioma [5]. In all, 23 patients with advanced angiosarcoma were recruited, and in these patients, the response rate was $9 \%(2 / 23) ; 43 \%(10 / 23)$ of patients progressed within 6 weeks, and the median PFS was 3 months.

The addition of bevacizumab ( $10 \mathrm{mg} / \mathrm{kg} 2$ weekly) to weekly paclitaxel $\left(90 \mathrm{mg} / \mathrm{m}^{2}\right)$ was studied in a noncomparative, open-label, randomised phase II study [6]. The response rate was lower with combination therapy (29\% [7/25] vs 46\% [11/24]), but the 6 month PFS rate was identical. Furthermore, the addition of bevacizumab to paclitaxel was associated with higher rates of serious toxicity (44\% vs 22\%), and more patients receiving combination treatment required dose reductions (56\% vs 35\%). The addition of bevacizumab to weekly paclitaxel is therefore not recommended.

\section{Oral tyrosine kinase inhibitors}

Angiosarcoma response to oral tyrosine kinase inhibitors that target VEGF receptors (VEGFR) has been studied in several phase II studies.

Tumour response to sorafenib (a VEGFR, Raf-1, and B-Raf inhibitor) $400 \mathrm{mg}$ bis die (bd) was reported in a study of 122 advanced sarcoma patients stratified by histological subtype [7]. One CR and 4 PR were observed in 34 patients with angiosarcoma. The median PFS of the vascular sarcoma cohort, which included 2 patients with solitary fibrous tumour and 1 patient with giant haemangioma, was 3.8 months. In comparison, the median PFS across all sarcoma subtypes was 3.2 months. Angiosarcoma response to sorafenib $400 \mathrm{mg}$ bd was studied by the French Sarcoma Group in two patient cohorts: 26 patients with cutaneous angiosarcoma and 15 patients with visceral disease [8]. The primary endpoint for this study was the 9-month progression-free rate. Only 2 patients, 1 from each cohort, were progression free at 9 months. The median PFS was 1.8 and 3.8 months for cutaneous and visceral disease respectively.

STS response to pazopanib (a VEGFR, fibroblast growth factor receptor [FGFR] and platelet derived growth factor receptor [PDGFR] inhibitor) $800 \mathrm{mg}$ omni die (od) was studied in the phase II EORTC 62043 [9], and subsequent randomised placebo-controlled phase III EORTC 62072 (PALETTE) study [10]. The median PFS of patients that received pazopanib in the PALETTE study was 4.6 months, compared to 1.6 months in patients that received placebo (HR 0.31 , 95\% CI 0.24-0.40; $p<0.0001)$. Angiosarcoma response to pazopanib was not reported separately; however a retrospective analysis of a cohort of 40 angiosarcoma patients treated with pazopanib in either the EORTC 62043 study, the PALETTE study, or in routine clinical practice, reported a $20 \%$ response rate and median PFS of 3.0 months [11]. There were no significant differences observed between different subtypes of angiosarcoma in response to pazopanib.

Angiosarcoma response to axitinib (a VEGFR, PDGFR and KIT inhibitor) $5 \mathrm{mg}$ bd was recently studied within a UK histologically stratified phase II study (NCT01140737), which also included cohorts for patients with advanced leiomyosarcoma, synovial sarcoma and 'other' STS histologies. Preliminary results from the angiosarcoma cohort were reported at the Connective Tissue Oncology Society 2016 annual meeting; the response rate was $10 \%, 12$ week nonprogression rate $42 \%$, and median PFS 4.2 months [12].

\section{Agents targeting non-VEGF angiogenic pathways}

\section{Angiopoietin 1/2}

Angiosarcoma response to weekly trebananib (30 mg/ kg), an angiopoietin-1 and -2 peptibody, was studied in a small single arm phase II study. No responses were observed and the median PFS was 1.6 months. STS response to regorafenib $160 \mathrm{mg}$ od, a multi-kinase inhibitor targeting VEGFR, PDGFR, Kit, RET and Raf-1, as well as TIE2, an angiopoietin receptor, was studied in a histologically stratified, randomised, placebo-controlled phase II study [13]. Similar to STS response to pazopanib, an unplanned pooled analysis of non-adipocytic STS cohorts reported a median PFS 4.0 months with regorafenib compared to 1.0 months with placebo (HR 0.36, 95\% CI $0.25-0.53 ; p<0.0001)$. Angiosarcoma response to regorafenib was not reported, but a separate study of regorafenib for advanced angiosarcoma is currently ongoing (NCT02048722).

\section{PDGF}

In addition to VEGFR, pazopanib, regorafenib and axitinib also inhibit PDGFR. Olaratumab is a humanised monoclonal antibody to PDGFa. Promising results were observed in a randomised phase I/II study of olaratumab in combination with doxorubicin as firstline treatment for advanced STS [14]; a phase III study recently completed recruitment and results are ea- 
gerly awaited (NCT 02451943). Angiosarcoma response to olaratumab has not been reported.

\section{Endoglin}

The transforming growth factor (TGF) $-\beta$ and bone morphogenetic protein (BMP)-9 receptors activin receptor-line kinase (ALK)-1 and endoglin are required for normal vascular development, and are highly expressed on tumour endothelial cells [15]. TRC105 is a chimeric monoclonal antibody that binds endoglin [16]. Non-adipocytic STS response to TRC105 in combination with pazopanib was studied in a phase I/II study [17]; response to treatment was observed in 8/9 angiosarcoma patients, including two durable CR. A randomised phase III trial of pazopanib \pm TRC105 in angiosarcoma recently opened to recruitment (NCT02979899).

\section{Other vascular targeted agents}

\section{Thalidomide}

Angiosarcoma response to thalidomide, an immunomodulatory agent with anti-angiogenic properties [18], has been described in several case reports $[19,20]$, however response has not been studied in a prospective trial.

\section{Metronomic chemotherapy}

Metronomic chemotherapy is the concept of administering chemotherapy drugs in frequent, often daily, low doses. It reduces tumour micro-vessel density, tumour hypoxia, and improves tumour perfusion, consistent with a vascular-targeted response [21]. Angiosarcoma response to metronomic chemotherapy has been reported in a few case studies [22]. A retrospective series of 26 elderly patients with advanced STS treated with metronomic oral cyclophosphamide $100 \mathrm{mg}$ bd, which included 3 patients with angiosarcoma, reported a disease control rate of $69 \%$ and a median PFS of 6.8 months [23].

\section{Beta-blockers}

The beta-blocker propranolol is recommended for the treatment of complex infantile haemangiomas [24]. Interestingly, beta-adrenergic receptors have also been identified as a potential therapeutic target in malignant vascular tumours [25]. A case series of 7 patients with advanced angiosarcoma treated with oral propranolol $40 \mathrm{mg}$ bd in combination with weekly metronomic vinblastine $\left(6 \mathrm{mg} / \mathrm{m}^{2}\right)$ plus methotrexate $\left(35 \mathrm{mg} / \mathrm{m}^{2}\right)$ reported a $100 \%$ response rate and a median PFS of 11 months [26].

\section{Discussion}

Despite their vascular nature, angiosarcoma response to VEGF-targeted agents is limited, and similar to the response observed in other more common STS subtypes. Biomarkers are required to identify patients most likely to respond to treatment. In a small cohort study, activating VEGFR2 mutations were identified in $14 \%(3 / 22)$ of angiosarcoma tumour samples [27]. No VEGFR2 mutations were identified in 27 tumour samples available for analysis from the French Sarcoma Group study of sorafenib for advanced angiosarcoma [8]. In a separate study, mutations in angiogenesisrelated genes were identified in $38 \%$ (15/39) of angiosarcoma tumour samples [28], including recurrent mutations in vascular endothelial-phosphotyrosine phosphatase (VE-PTP) and phospholipase gamma 1 (PLCG1). The response of angiosarcomas harbouring mutations in angiogenesis-related genes to vascular targeted agents has not been correlated.

Angiosarcoma tumour cells express multiple angiogenic growth factors [29]. Angiosarcoma expression of these factors is associated with improved patient outcomes [30], and thus expression may be a measure of tumour endothelial differentiation rather than a pathological driver of disease. Furthermore, the expression of multiple angiogenic growth factors represents potential resistance mechanisms to targeted agents. Despite the negative results of paclitaxel in combination with bevacizumab [6], further combination studies-either anti-angiogenic agents in combination with chemotherapy or combinations of different anti-angiogenic agents-are indicated.

Conflict of interest R.J. Young and P.J. Woll declare that they have no competing interests.

Open Access This article is distributed under the terms of the Creative Commons Attribution 4.0 International License (http://creativecommons.org/licenses/by/4.0/), which permits unrestricted use, distribution, and reproduction in any medium, provided you give appropriate credit to the original author(s) and the source, provide a link to the Creative Commons license, and indicate if changes were made.

\section{References}

1. Young RJ, Brown NJ, Reed MW, Hughes D, Woll PJ. Angiosarcoma. Lancet Oncol. 2010;11:983-91.

2. Belotti D, Vergani V, Drudis T, Borsotti P, Pitelli MR, Viale $\mathrm{G}$, et al. The microtubule-affecting drug paclitaxel has antiangiogenic activity. Clin Cancer Res. 1996;2:1843-9.

3. PenelN, BuiBN, BayJO, CupissolD, Ray-CoquardI, PipernoNeumann S, et al. Phase II trial of weekly paclitaxel for unresectable angiosarcoma: the ANGIOTAX Study. J Clin Oncol. 2008;26:5269-74.

4. Young RJ, Natukunda A, Litière S, Woll PJ, Wardelmann E, van der Graaf WT. First-line anthracycline-based chemotherapy for angiosarcoma and other soft tissue sarcoma subtypes: pooled analysis of eleven European Organisation for Research and Treatment of Cancer Soft 
Tissue and Bone Sarcoma Group trials. Eur J Cancer. 2014;50:3178-86.

5. Agulnik M, Yarber JL, Okuno SH, von Mehren M, Jovanovic BD, Brockstein BE, et al. An open-label, multicenter, phase II study of bevacizumab for the treatment of angiosarcoma and epithelioid hemangioendotheliomas. Ann Oncol. 2013;24:257-63.

6. Ray-Coquard IL, Domont J, Tresch-Bruneel E, Bompas E, Cassier PA, Mir O, et al. Paclitaxel given once per week with or without bevacizumab in patients with advanced angiosarcoma: a randomized phase II trial. J Clin Oncol. 2015;33:2797-802.

7. Maki RG, D'Adamo DR, Keohan ML, Saulle M, Schuetze SM, Undevia SD, et al. Phase II study of sorafenib in patients with metastatic or recurrent sarcomas. J Clin Oncol. 2009;27:3133-40.

8. Ray-Coquard I, Italiano A, Bompas E, Le CesneA, Robin YM, Chevreau C, et al. Sorafenib for patients with advanced angiosarcoma: a phase II Trial from the French Sarcoma Group (GSF/GETO). Oncologist. 2012;17:260-6.

9. Sleijfer S, Ray-Coquard I, Papai Z, Le Cesne A, Scurr M, Schöffski P, et al. Pazopanib, a multikinase angiogenesis inhibitor, in patients with relapsed or refractory advanced soft tissue sarcoma: a phase II study from the European organisation for research and treatment of cancer-soft tissue and bone sarcoma group (EORTC study 62043). J Clin Oncol. 2009;27:3126-32.

10. van der Graaf WT, Blay JY, Chawla SP, Kim DW, Bui-Nguyen B, Casali PG, et al. Pazopanib for metastatic soft-tissue sarcoma (PALETTE): a randomised, double-blind, placebocontrolled phase 3 trial. Lancet. 2012;379:1879-86.

11. Kollár A, Jones RL, Stacchiotti S, Gelderblom H, Guida M, GrignaniG, etal. Pazopanibin advanced vascular sarcomas: an EORTC Soft Tissue and Bone Sarcoma Group (STBSG) retrospective analysis. Acta Oncol. 2017;56:88-92.

12. Penella W, Piers G, Ian J, Sandra S, Jeremy W, Michael L, et al. AXI-STS: A UK NCRI Sarcoma Clinical Studies Group phase II trial of the VEGFR inhibitor axitinib in advanced soft tissue sarcoma (STS). Poster session presented at: $21^{\text {st }}$ Connective Tissue Oncology Society Annual Meeting; 9-12. Nov; Lisbon. 2016. p. 2554618.

13. Mir O, Brodowicz T, Italiano A, Wallet J, Blay JY, Bertucci F, et al. Safety and efficacy of regorafenib in patients with advanced soft tissue sarcoma (REGOSARC): a randomised, double-blind, placebo-controlled, phase 2 trial. Lancet Oncol. 2016;17:1732-42.

14. Tap WD, Jones RL, Van Tine BA, Chmielowski B, Elias AD, Adkins D, et al. Olaratumab and doxorubicin versus doxorubicin alone for treatment of soft-tissue sarcoma: an open-label phase $1 \mathrm{~b}$ and randomised phase 2 trial. Lancet. 2016;388:488-97.

15. Jonker L. TGF- $\beta$ \& BMP receptors endoglin and ALK1: overview of their functional role and status as antiangiogenic targets. Microcirculation. 2014;21:93-103.

16. Rosen LS, Hurwitz HI, Wong MK, Goldman J, Mendelson DS, FiggWD, et al. A phase Ifirst-in-human study of TRC105 (Anti-EndoglinAntibody) in patients with advanced cancer. Clin Cancer Res. 2012;18:4820-9.

17. Attia S, Sankhala KK, Riedel RF, Robinson SI, Conry RM, Boland PM, et al. A phase 1B/phase 2A study of TRC105 (Endoglin Antibody) in combination with pazopanib $(\mathrm{P})$ in patients (pts) with advanced soft tissue sarcoma (STS).JClin Oncol. 2016;34:11016.

18. Zhou S, Wang F, Hsieh TC, Wu JM, Wu E. Thalidomide-a notorious sedative to a wonder anticancer drug. Curr Med Chem. 2013;20:4102-8.

19. Raina V, Sengar M, Shukla NK, Deo SS, Mohanty BK, Sharma $\mathrm{D}$, et al. Complete response from thalidomide in angiosarcoma after treatment of breast cancer. J Clin Oncol. 2007;25:900-1.

20. Alvarado-Miranda A, Bacon-Fonseca L, Ulises Lara-Medina F, Maldonado-Martínez H, Arce-Salinas C. Thalidomide combined with neoadjuvant chemotherapy in angiosarcoma of the breast with complete pathologic response: case report and review of literature. Breast Care (Basel). 2013;8:74-6.

21. Penel N, Adenis A, Bocci G. Cyclophosphamide-based metronomic chemotherapy: after 10 years of experience, where do we stand and where are we going? Crit Rev Oncol Hematol. 2012;82:40-50.

22. Wong H, Yau T. Review: metronomic chemotherapy for advanced cutaneous angiosarcoma. Clin Adv Hematol Oncol. 2011;9:504-6.

23. Mir O, Domont J, Cioffi A, Bonvalot S, Boulet B, Le Pechoux $\mathrm{C}$, et al. Feasibility of metronomic oral cyclophosphamide plus prednisolone in elderly patients with inoperable or metastatic soft tissue sarcoma. Eur JCancer. 2011;47:515-9.

24. Léauté-Labrèze C, Harper JI, Hoeger PH. Infantile haemangioma. Lancet. 2017;390:85-94.

25. Stiles JM, Amaya C, Rains S, Diaz D, Pham R, Battiste J, et al. Targeting of beta adrenergic receptors results in therapeutic efficacy against models of hemangioendothelioma and angiosarcoma. PLOS ONE. 2013;8:e60021.

26. PasquierE,AndréN, StreetJ, ChouguleA, RekhiB, GhoshJ, et al. Effective management of advanced angiosarcoma by the synergistic combination of propranolol and vinblastinebased metronomic chemotherapy: a bench to bedside study. EBioMedicine. 2016;6:87-95.

27. Antonescu CR, Yoshida A, Guo T, Chang NE, Zhang L, Agaram NP, et al. KDR activating mutations in human angiosarcomas are sensitive to specific kinase inhibitors. Cancer Res. 2009;69:7175-9.

28. Behjati S, Tarpey PS, Sheldon H, Martincorena I, Van Loo P, Gundem G, etal. Recurrent PTPRB and PLCG1 mutations in angiosarcoma. Nat Genet. 2014;46(4):376. https://doi.org/ $10.1038 / \mathrm{ng} .2921$.

29. Young RJ, Fernando M, Hughes D, Brown NJ, Woll PJ. Angiogenic growth factor expression in benign and malignant vascular tumours. Exp Mol Pathol. 2014;97:148-53.

30. Itakura E, Yamamoto H, Oda Y, Tsuneyoshi M. Detection and characterization of vascular endothelial growth factors and their receptors in a series of angiosarcomas. J Surg Oncol. 2008;97:74-81.

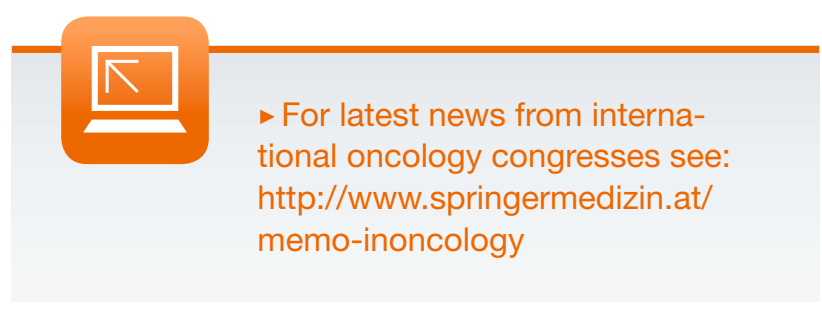

